This article is licensed under the Creative Commons Attribution-NonCommercial 4.0 International License (CC BY-NC) (http://www.karger.com/Services/OpenAccessLicense). Usage and distribution for commercial purposes requires written permission.

\title{
Epstein-Barr Virus-Associated Encephalopathy Presenting with Nonconvulsive Status Epilepticus in an Immunosuppressive State
}

\author{
Yuichiro Ohya $^{a} \quad$ Kuniyuki Nakamura $^{a}$ Yoshinobu Wakisaka ${ }^{a}$ \\ Hiroaki Sato $^{a}$ Kayo Wakisaka ${ }^{a}$ Masaya Kumamoto ${ }^{a}$ Yohei Muraya $^{b}$ \\ Junya Kuroda ${ }^{b}$ Hiroshi Nakane ${ }^{b}$ Goichi Yoshimoto $^{c}$ \\ Takanari Kitazono ${ }^{a}$ Tetsuro Ago ${ }^{a}$ \\ aDepartment of Medicine and Clinical Science, Graduate School of Medical Sciences, \\ Kyushu University, Fukuoka, Japan; ${ }^{b}$ Cerebrovascular and Neurology Center, National \\ Hospital Organization Fukuoka-Higashi Medical Center, Koga, Japan; 'Department of \\ Medicine and Biosystemic Science, Graduate School of Medical Sciences, Kyushu \\ University, Fukuoka, Japan
}

\section{Keywords}

Epstein-Barr virus · Encephalopathy · Epilepsy · Hyperperfusion · Nonconvulsive status epilepticus

\section{Abstract}

Epstein-Barr virus (EBV) infection is occasionally accompanied by central nervous system (CNS) complications, particularly in immunosuppressed patients. However, the symptoms and clinical features of EBV infection in the CNS are rather heterogeneous and remain unknown. We herein describe the first reported adult case manifesting nonconvulsive status epilepticus (NCSE), possibly associated with reactivation of EBV in an immunosuppressive state. A 63-year-old man with a history of acute myeloid leukemia and taking immunosuppressants was admitted due 


\section{Case Reports in Gastroenterology}

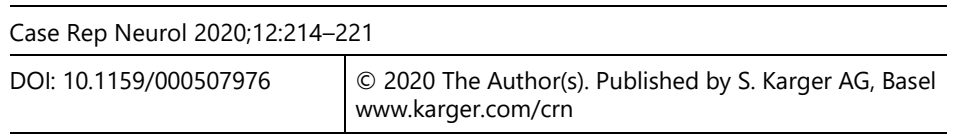

Ohya et al.: EBV-Associated Encephalopathy Presenting with NCSE

to progressively impaired consciousness without any focal neurological signs, including paralysis or convulsions. Arterial spin labeling magnetic resonance imaging (ASL-MRI) and brain perfusion single-photon emission computed tomography showed hyperperfusion in the right temporal region, despite no morphological abnormalities in other MRI sequences. White blood cell counts, EBV viral load, and virus-capsid antigen IgG in cerebrospinal fluid were elevated. We diagnosed him with EBV-associated encephalopathy presenting with NCSE. Administration of levetiracetam, an antiepileptic, improved the consciousness and the abnormal hyperperfusion. This case suggests a new concept of EBV-associated encephalopathy leading to epilepsy, particularly in immunosuppressed patients.

(c) 2020 The Author(s)

Published by S. Karger AG, Basel

\section{Introduction}

Epstein-Barr virus (EBV) infects over $90 \%$ of the adult population worldwide. EBV infection usually occurs early in childhood and produces no symptoms. By contrast, delayed primary infection during adolescence can lead to infectious mononucleosis [1]. EBV primarily targets and remains latent in memory B cells. Reactivation of latent EBV is often found in immunosuppressive states, and is clinically important because it causes lymphoproliferative disease and central nervous system (CNS) complications [2]. CNS symptoms, including encephalitis, meningitis, cerebellitis, polyradiculomyelitis, transverse myelitis, cranial and peripheral neuropathies, and psychiatric abnormalities, can occur in 1-18\% of patients with EBV infection [2]. EBV-associated encephalopathy/encephalitis often presents with nonspecific symptoms and its diagnosis is based on the detection of EBV antibodies and/or DNA in the cerebrospinal fluid (CSF). The clinical features and pathophysiology of EBV infection in the CNS in adulthood remain unknown.

Herein, we describe an immunosuppressed adult male with EBV infection in the CNS, presenting with nonconvulsive status epilepticus (NCSE), revealed by arterial spin labeling magnetic resonance imaging (ASL-MRI) and single-photon emission computed tomography (SPECT).

\section{Case Presentation}

A 63-year-old man was admitted to our hospital due to gradually impaired consciousness. He had been diagnosed with acute myeloid leukemia at 59 years of age. Remission of leukemia was achieved after allogeneic hematopoietic stem cell transplantation, and consequently he was treated with prednisolone ( $2 \mathrm{mg} /$ day) and tacrolimus $(0.2 \mathrm{mg} /$ day). About 1 week before admission to our hospital, he presented with a slight fever and malaise. He became progressively unable to walk and talk. Subsequently, his consciousness level was impaired to 11 points (E3, V3, M5) on the Glasgow Coma Scale (GCS), and he was admitted to our hospital. One day after admission, his consciousness level further decreased to 9 points (E2, V2, M5) on the GCS. His vital signs (i.e., blood pressure, pulse, and body temperature) were normal. There were no physical findings of neck stiffness, lymphadenopathy, rash, hepatosplenomegaly, or jaundice. The impaired consciousness was not accompanied by paralysis or convulsions. 


\section{Case Reports in Gastroenterology}

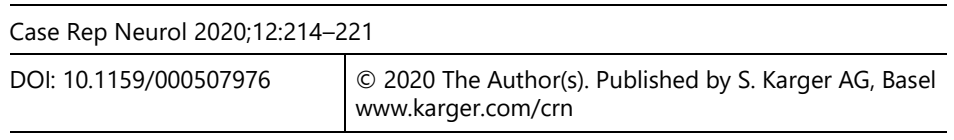

Ohya et al.: EBV-Associated Encephalopathy Presenting with NCSE

Blood tests for factors that might cause impaired consciousness, such as inflammation, electrolyte imbalance, hypo/hyperglycemia, and uremia, were normal (Table 1). Fluid-attenuated inversion recovery (FLAIR) and diffusion-weighted imaging (DWI) of brain MRI revealed no abnormalities (Fig. 1a-d). Magnetic resonance angiography also revealed no abnormalities during hospitalization (Fig. 1e, f). However, ASL-MRI and brain perfusion SPECT showed cerebral hyperperfusion in the right temporal region (Fig. 2a, b). Apart from generalized slower theta or delta frequencies, wakeful electroencephalography revealed no abnormal findings that would indicate epilepsy. Examination of CSF showed slight increases in white blood cell count $(14$ cells $/ \mu \mathrm{L})$, protein $(73.6 \mathrm{mg} / \mathrm{dL})$, and glucose $(62.0 \mathrm{mg} / \mathrm{dL})$. CSF cytology found no malignant cells. EBV virus capsid antigen (VCA) immunoglobulin (Ig)M was negative in CSF, yet EBV VCA IgG was positive. Quantitative PCR revealed that EBV DNA in CSF was increased to 720 copies/mL, suggesting EBV reactivation in the CNS. In contrast, DNA of herpes virus type 1 or cytomegalovirus was not detected by PCR.

Based on the findings of MRI and EBV DNA in CSF, we diagnosed him with EBV-associated encephalopathy with mild inflammation. Administration of an antiepileptic, levetiracetam $(1,000 \mathrm{mg} /$ day) promptly improved his consciousness to 15 points (E4, V5, M6) on the GCS. He regained the ability to stand and walk without any support on the 14th hospital day. SPECT showed disappearance of hyperperfusion in the temporal region on the 17th day (Fig. 2c). After discharge, the dosage of tacrolimus was decreased from $0.2-0.1 \mathrm{mg} / \mathrm{day}$.

\section{Discussion/Conclusion}

We herein report a late middle-aged man who developed NCSE with focal hyperperfusion. Because he was in an immunosuppressive state and we detected significant amounts of EBV DNA and elevated EBV VCA IgG in CSF [2], we speculate that immunosuppression-associated reactivation of EBV may have caused encephalopathy leading to NCSE with mild inflammation. To the best of our knowledge, this is the first reported adult case of EBV-associated encephalopathy presenting with NCSE.

An obvious abnormal finding related to impaired consciousness in the present case was focal hyperperfusion detected by ASL-MRI and SPECT, and which is a characteristic of focal epilepsy [3-6]. In addition, the fact that the administration of levetiracetam promptly improved both consciousness disturbance and focal hyperperfusion supported our diagnosis of NCSE in this case. Even so, repeated electroencephalography did not identify any obvious epileptic spikes.

The symptoms and clinical features of EBV infection in the CNS are heterogeneous and may be dependent upon the degree of cellular dysfunction or destruction, i.e., from encephalopathy to encephalitis. There are two reported pediatric cases with NCSE due to EBV encephalitis $[7,8]$. Both cases exhibited hyperintensity lesions on T2-weighted images, while EBV VCA IgM was positive, indicating primary infection of EBV. Abnormal brain MRI findings suggest that primary infection of EBV during pediatric periods may cause destructive neural damage accompanied by severe inflammation. In contrast, Park et al. [9] reported a case of a 30year-old woman with EBV cerebellitis, who had no prior history of illness. This case showed hyperperfusion in both cerebellar hemispheres on SPECT without any abnormalities on MRI. Moreover, she recovered fully without any neurological sequelae, similar to our case. In our 


\section{Case Reports in Gastroenterology}

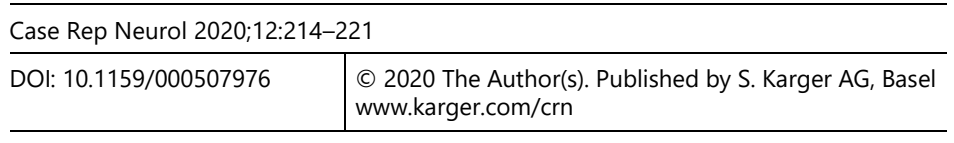

Ohya et al.: EBV-Associated Encephalopathy Presenting with NCSE

present case, reactivation of EBV may have caused neural dysfunction leading to NCSE with mild inflammation.

Usefulness of an antiviral or immunomodulatory treatment for EBV infection has not yet been established. The above two cases were treated with acyclovir [7, 8] and corticosteroid [8]. Meanwhile, both the case of Park et al. [9] and our case showed hyperperfusion without any morphological abnormalities and improved with only supportive therapies. The degree of inflammation may provide a good indication on how to treat these patients.

While EBV typically infects and remains in memory B cells in the latent phase [10] (in which EBV remains "hidden" in healthy hosts), EBV DNA is often detected in CSF in patients under immunosuppressive conditions [2]. In our present case, continuous treatment with prednisolone and tacrolimus after stem cell transplantation may have caused latent infection and reactivation of EBV in the CNS. EBV can enter B cells by binding to gp350 and complement receptor 2 (CR2), which are highly expressed on the plasma membranes of $B$ cells [10]. Because CR2 is also highly expressed in astrocytes in the CNS $[1,11]$, EBV can also enter astrocytes with high affinity and remain latent [12], ultimately causing astrocytic dysfunction. It is also suggested that some astrocytoma cases are associated with EBV infection [13]. A possible pathophysiological mechanism of the present case may be as follows: initially, EBV was infected and reactivated in astrocytes in an immunosuppressive state. Next, the dysfunction of astrocytes may have caused neuronal hyperexcitability leading to epilepsy with cerebral hyperperfusion $[6,14,15]$.

In conclusion, we report an interesting case with immunosuppression-associated reactivation of EBV in the CNS that may possibly cause NCSE accompanied with focal hyperperfusion and mild inflammation. This case suggests a new concept of EBV-associated encephalopathy leading to epilepsy, particularly in immunosuppressive patients and including aged people. Further investigations to uncover the pathophysiology of EBV-associated encephalopathy may be needed in future.

\section{Acknowledgments}

We thank Rachel James, PhD, from the Edanz Group (www. edanzediting.com) for English editing.

\section{Statement of Ethics}

There are no ethical conflicts to declare. The patient was informed of the purpose of the case presentation, and provided written informed consent for the publication, including images, case history, and clinical data.

\section{Disclosure Statement}

The authors have no conflicts of interest to declare. 


\section{Case Reports in Gastroenterology}

\section{Funding Sources}

The authors have no funding sources to declare.

\section{Author Contributions}

All authors read and approved the manuscript before submission. Yuichiro Ohya and Kuniyuki Nakamura reviewed the clinical data, performed the literature search, and drafted the manuscript. Yoshinobu Wakisaka, Hiroaki Sato, Kayo Wakisaka, Masaya Kumamoto, Yohei Muraya, Junya Kuroda, Hiroshi Nakane, Goichi Yoshimoto, Takanari Kitazono, and Tetsuro Ago contributed to the diagnosis and revised the manuscript.

\section{References}

1 Hassani A, Corboy JR, Al-Salam S, Khan G. Epstein-Barr virus is present in the brain of most cases of multiple sclerosis and may engage more than just B cells. PLoS One. 2018 Feb;13(2):e0192109.

2 Martelius T, Lappalainen M, Palomäki M, Anttila VJ. Clinical characteristics of patients with Epstein Barr virus in cerebrospinal fluid. BMC Infect Dis. 2011 Oct;11(1):281.

3 Shimogawa T, Morioka T, Sayama T, Haga S, Kanazawa Y, Murao K, et al. The initial use of arterial spin labeling perfusion and diffusion-weighted magnetic resonance images in the diagnosis of nonconvulsive partial status epileptics. Epilepsy Res. 2017 Jan;129:162-73.

4 Rowe CC, Berkovic SF, Sia ST, Austin M, McKay WJ, Kalnins RM, et al. Localization of epileptic foci with postictal single photon emission computed tomography. Ann Neurol. 1989 Nov;26(5):660-8.

5 Schwartz R, Jones K, Kalina P, Bajakian R, Mantello M, Garada B, et al. Hypertensive encephalopathy: findings on CT, MR imaging, and SPECT imaging in 14 cases. Am J Roentgenol. 1992 Aug;26(5):379-83.

6 Iizuka T, Sakai F. Pathogenesis of stroke-like episodes in MELAS: analysis of neurovascular cellular mechanisms. Curr Neurovasc Res. 2005 Jan;2(1):29-45.

7 Greco F, Cocuzza MD, Smilari P, Sorge G, Pavone L. Nonconvulsive status epilepticus complicating epsteinbarr virus encephalitis in a child. Case Rep Pediatr. 2014;2014:547396.

8 Rodrigo-Armenteros P, Kapetanovic-García S, Antón-Méndez L, Gómez-Muga JJ, Río EB, Fernández-Cuesta MA, et al. Akinetic mutism and status epilepticus due to Epstein Barr virus encephalitis. Clin Neurol Neurosurg. 2019 Oct;185:105492.

9 Park JW, Choi YB, Lee KS. Detection of acute Epstein Barr virus cerebellitis using sequential brain HMPAOSPECT imaging. Clin Neurol Neurosurg. 2004 Mar;106(2):118-21.

10 Mui UN, Haley CT, Tyring SK. Viral Oncology: Molecular Biology and Pathogenesis. J Clin Med. 2017 Nov;6(12):E111.

11 Gasque P, Chan P, Mauger C, Schouft MT, Singhrao S, Dierich MP, et al. Identification and characterization of complement C3 receptors on human astrocytes. J Immunol. 1996 Mar;156(6):2247-55.

12 Menet A, Speth C, Larcher C, Prodinger WM, Schwendinger MG, Chan P, et al. Epstein-Barr virus infection of human astrocyte cell lines. J Virol. 1999 Sep;73(9):7722-33.

13 Akhtar S, Vranic S, Cyprian FS, Al Moustafa AE. Epstein-Barr virus in gliomas: cause, association, or artifact? Front Oncol. 2018 Apr;8:123.

14 DiFrancesco JC, Cooper JM, Lam A, Hart PE, Tremolizzo L, Ferrarese C, et al. MELAS mitochondrial DNA mutation A3243G reduces glutamate transport in cybrids cell lines. Exp Neurol. 2008 Jul;212(1):152-6.

15 Attwell D, Buchan AM, Charpak S, Lauritzen M, Macvicar BA, Newman EA. Glial and neuronal control of brain blood flow. Nature. 2010 Nov;468(7321):232-43. 


\section{Case Reports in Gastroenterology}

Case Rep Neurol 2020;12:214-22

DOI: $10.1159 / 000507976$

(C) 2020 The Author(s). Published by S. Karger AG, Basel

Ohya et al.: EBV-Associated Encephalopathy Presenting with NCSE

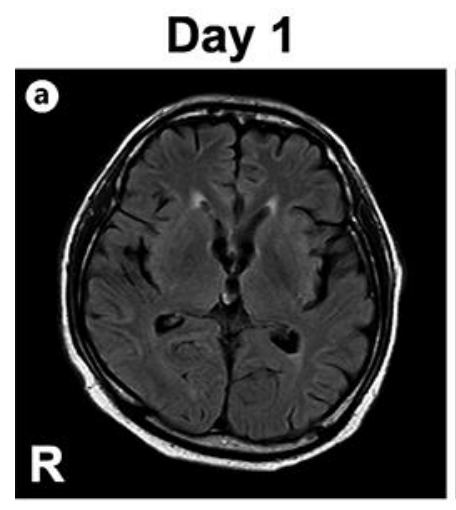

Day 4

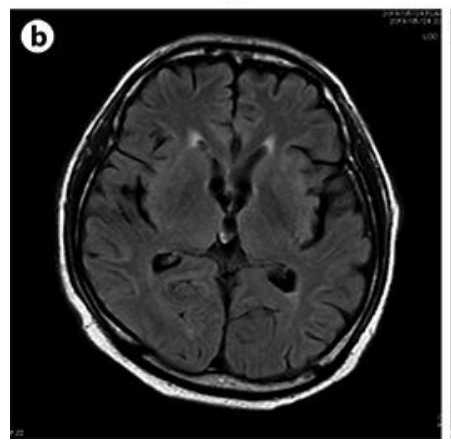

Day 1

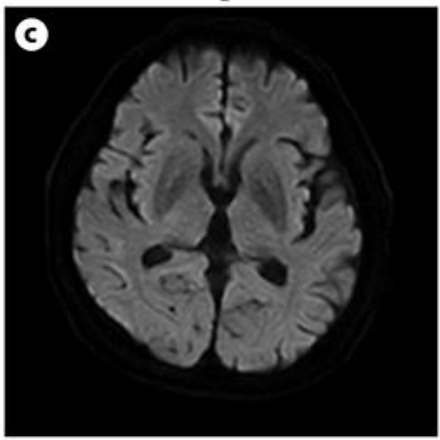

Day 4

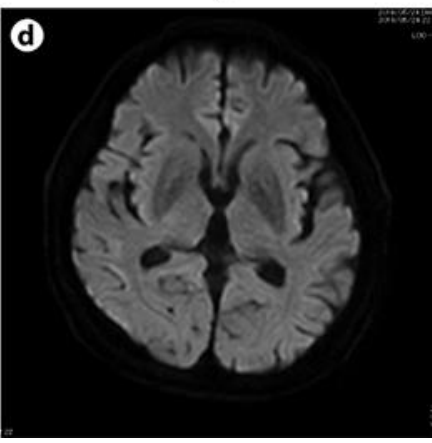

Day 1

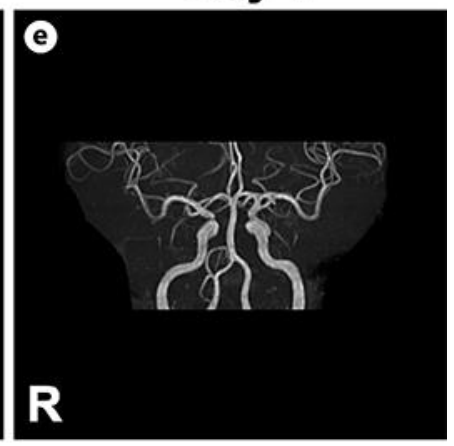

Day 4

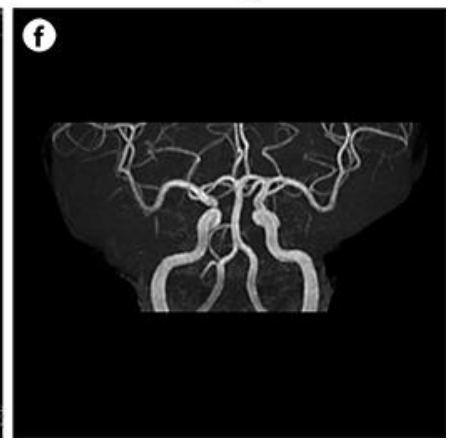

Fig. 1. Brain magnetic resonance imaging (MRI) and magnetic resonance angiography (MRA). Fluid-attenuated inversion recovery (FLAIR) (a, b) and diffusion-weighted imaging (DWI) (c, d) of brain MRI at 1 day or 4 days after admission to our hospital revealed no abnormalities related to decreased consciousness. MRA revealed no abnormalities at 1 day (e) or 4 days (f) after admission. "R" indicates right side. 


\section{Case Reports in Gastroenterology}

Case Rep Neurol 2020;12:214-221

DOI: $10.1159 / 000507976$

(C) 2020 The Author(s). Published by S. Karger AG, Basel www.karger.com/crn

Ohya et al.: EBV-Associated Encephalopathy Presenting with NCSE
Day 4

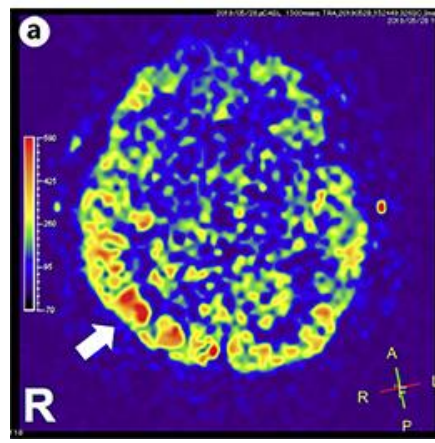

Day 5

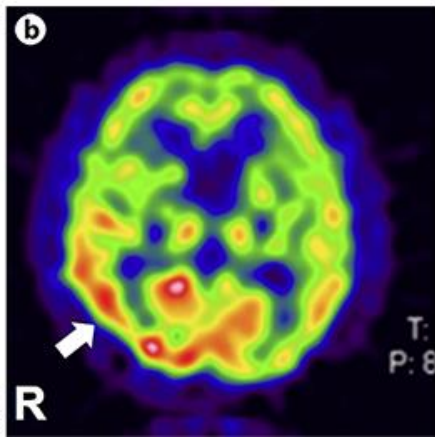

Day 17

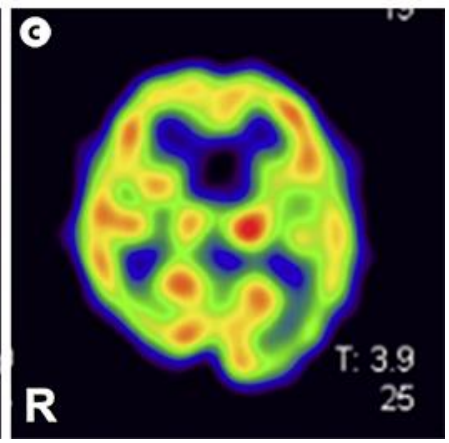

Fig. 2. Brain perfusion images. a Arterial spin labeling magnetic resonance imaging (ASL-MRI) showed cerebral hyperperfusion (arrow) in the right temporal region at 4 days after admission to our hospital. $\mathbf{b}$ Brain single-photon emission computed tomography (SPECT) showed hyperperfusion (arrow) in the right temporal region at 5 days after admission. c After administration of an antiepileptic levetiracetam, hyperperfusion by SPECT improved at 17 days after admission. "R" indicates right side. 
Case Reports in Gastroenterology
Case Rep Neurol 2020;12:214-221

\begin{tabular}{l|l}
\hline DOI: $10.1159 / 000507976$ & (C) 2020 The Author(s). Published by S. Karger AG, Basel
\end{tabular} www.karger.com/crn

Ohya et al.: EBV-Associated Encephalopathy Presenting with NCSE

Table 1. Results of peripheral blood and cerebrospinal fluid analysis

\begin{tabular}{ll}
\hline Test & Laboratory value \\
\hline Blood & \\
White blood cell count, / $\mu \mathrm{L}$ & 6,500 \\
Red blood cell count, / $\mu \mathrm{L}$ & $3,600,000$ \\
Hemoglobin, g/dL & 12.1 \\
Platelets, $n / \mu \mathrm{L}$ & 105,000 \\
C-reactive protein, mg/dL & 0.62 \\
AST, IU/L & 75 \\
ALT, IU/L & 88 \\
Blood urea nitrogen, mg/dL & 22 \\
Creatinine, mg/dL & 1.52 \\
Na, mmol/L & 142 \\
K, mmol/L & 4.6 \\
Glucose, mg/dL & 106 \\
Lactate, mg/dL & 9.7 \\
Pyruvate, mg/dL & 0.8 \\
EBNA & 20 \\
EBV VCA IgM & 0.3 \\
EBV VCA IgA & 40 \\
EBV VCA IgG & 11.7 \\
\hline Cerebrospinal fluid & \\
Cell count, / $\mu \mathrm{L}$ & 14 \\
Protein, mg/dL & 73 \\
Glucose, mg/dL & 62 \\
HSV I PCR, copies/mL & $<100$ \\
CMV PCR, copies/mL & 100 \\
EBV PCR, copies/mL & 32 \\
EBV VCA IgG & 1 \\
EBV VCA IgM & \\
Lactate, mg/dL & \\
Pyruvate, mg/dL & \\
\hline
\end{tabular}

